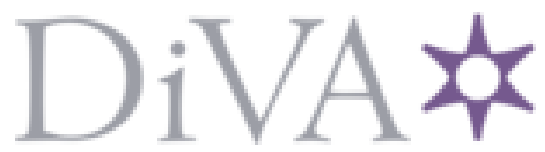

http://www.diva-portal.org

\title{
Preprint
}

This is the submitted version of a paper presented at 58th IEEE Global Communications Conference, GLOBECOM 2015, San Diego, United States, 6 December 2015 through 10 December 2015.

Citation for the original published paper:

Loomba, R., de Fréin, R., Brendan, J. (2016)

Selecting Energy Efficient Cluser-Head Trajectories for Collaborative Mobile Sensing. In: IEEE (ed.), 2015 IEEE Global Communications Conference, GLOBECOM 2015 Institute of Electrical and Electronics Engineers (IEEE) http://dx.doi.org/10.1109/GLOCOM.2014.7417727

N.B. When citing this work, cite the original published paper.

Permanent link to this version:

http://urn.kb.se/resolve?urn=urn:nbn:se:kth:diva-173803 


\section{Selecting Energy Efficient Cluster-Head Trajectories for Collaborative Mobile Sensing}

Radhika Loomba ${ }^{\dagger \dagger}$ and Ruairí de Fréin ${ }^{\dagger}$ and Brendan Jennings ${ }^{\dagger \dagger}$

${ }^{\dagger} \mathrm{KTH}$ - Royal Institute of Technology, Stockholm, Sweden

${ }^{\dagger}$ Waterford Institute of Technology, Ireland

web: https://robustandscalable.wordpress.com

in: IEEE Global Communications Conference (GLOBECOM). See also BIBT $\mathrm{X}$ entry below.

BIBTEX:

darticle\{rdefrein15Globecom,

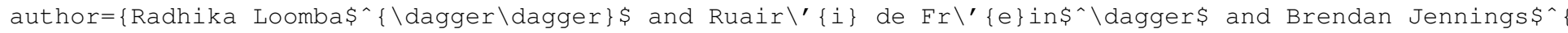
journal=\{IEEE Global Communications Conference (GLOBECOM) $\}$,

title=\{Selecting Energy Efficient Cluster-Head Trajectories for Collaborative Mobile Sensing\}, year $=\{2015\}$,

pages $=\{7\}$,

month $=\{$ Dec $\}$,

(C) 2015 IEEE. Personal use of this material is permitted. However, permission to reprint/republish this material for advertising or promotional purposes or for creating new collective works for resale or redistribution to servers or lists, or to reuse any copyrighted component of this work in other works must be obtained from the IEEE. 


\title{
Selecting Energy Efficient Cluster-Head Trajectories for Collaborative Mobile Sensing
}

\author{
Radhika Loomba* and Ruairí de Fréin ${ }^{* \dagger}$ and Brendan Jennings* \\ *TSSG, Waterford Institute of Technology, Ireland \\ ${ }^{\dagger}$ KTH - Royal Institute of Technology, Stockholm, Sweden \\ Email: r.l.loomba@ieee.org, rdefrein@gmail.com, bjennings@tssg.org
}

\begin{abstract}
We study the design of energy-efficient stochastic leader-selection algorithms in environments which are sensed by mobile handsets. This work optimizes the energy usage of the interaction between mobile handsets and an application server. The energy-efficiency of mobile handset communications is of increasing importance given the convergence of mobile computing and cloud computing. In some scenarios the lack of $\mathrm{WiFi}$ forces mobile devices to expend additional energy transmitting application specific sensed data over cellular networks. This paper focuses on (1) fairly allocating the overhead of transmitting sensed data to a cellular base station amongst the mobile devices by selecting a cluster-head (Fairness is measured in terms of the relative energy expended); (2) successively improving the location of the selected leader such that the efficiency of the entire system is improved; and finally, (3) allowing the mobile devices to gradually forget what they have learned so that the life-time of the mobile devices is increased (without significantly affecting energy savings). We present two algorithms in this paper and demonstrate that the battery life-time associated with sensor data transmissions of the mobile handsets may be increased by $20-40 \%$ without incurring a sensing accuracy penalty.
\end{abstract}

\section{INTRODUCTION}

The proliferation of smart phones (mobiles in what follows) with increasingly sophisticated sensors provides an attractive platform for mobile sensing. Applications in the areas of health-care, social networks and environmental monitoring [17] harness this diverse sensor data for user personalization. These applications leverage mobile cloud computing technologies and allow dynamic offloading of the sensed data to address the resource and processing limitations of the mobile [26], [16].

Energy-efficiency is a major concern. In situations where WiFi is unavailable, mobiles expend additional energy when transmitting application specific sensed data over cellular networks. The alternative is that the mobiles do not report their sensor readings, and the accuracy of the system is affected. Existing energy-efficient techniques [4], [3], [15] aim to reduce the amount of offloaded data of an application to achieve a trade-off with the amount of energy expended. However, lossless data compression techniques are limited by the entropy of the sensor readings [8], and thus, other approaches are required to increase energy efficiency. Optimizing the selection of mobiles to offload this reduced data, is a promising approach, but also a challenging open problem. We propose a cluster-head selection algorithm that adapts the collaboration between mobiles -in close proximity- by optimizing the transmission costs: cost is measured in this paper in terms of the communication distance between the mobiles and the cellular Base Station (BS) and the inter-mobile communication distances. We envisage a system that exploits the fact that shorter transmit distances for communications are cheap, and that the few longer distance transmissions that are necessary -to offload aggregated sensed data to the Application Cloud Server (ACS) via the BS- can be optimized by using only suitably positioned Cluster-Heads $(\mathrm{CH})$ to perform these transmissions. The distribution of mobiles and the BS placement cannot be controlled; we can however optimize the selection of the $\mathrm{CH}$ for energy savings. In addition, the $\mathrm{CHs}$ must also be changed as time passes to ensure that all transmission costs are not incurred by one (a few) mobile(s) -the protocol should be fair. Sharing the transmission energy between the mobiles has the benefit of potentially extending the average lifetime of all mobiles; therefore, the proposed approach is timely and of interest to the mobile sensing community, in that it meets one of the technical challenges most likely arrest the wide-spread deployment of mobile sensing technology -battery-life.

This paper focuses on the deployment of a $\mathrm{CH}$ selection technique in open areas like a park, a shopping mall or a railway station. Mobiles collaborate to send sensing information about the temperature, humidity, gas etc. of the area to an ACS over the available cellular network. We treat the selection of the $\mathrm{CH}$ mobile as a separate problem from the data compression problem [9]. We contribute two algorithms that select $\mathrm{CHs}$ in an ad-hoc, low latency, bandwidth and energy manner. Knowledge of the communication distance between the mobiles and the cellular BS is used to modulate the probability of each mobile becoming a $\mathrm{CH}$ during the next sensing period: we exploit the notion of each CH's trajectory, e.g. the identity of the $\mathrm{CH}$ is improved from sensing period to sensing period by favourably biasing the probability that neighbouring mobiles become a $\mathrm{CH}$, if the current $\mathrm{CH}$ is well positioned. We compare our solutions with LEACH [13], a widely used cluster-based technique for wireless sensor networks. The contribution in this paper goes beyond that of LEACH in that we allow knowledge of good $\mathrm{CH}$ locations to feed-forward into future $\mathrm{CH}$ selection decisions. In terms of our simulation set-up, the COST-231 propagation model [5] is used to simulate a micro-cell deployment. Rhee et al. [24] have presented a truncated Levy walk model which provides a simple and realistic model for human mobility. This improved 


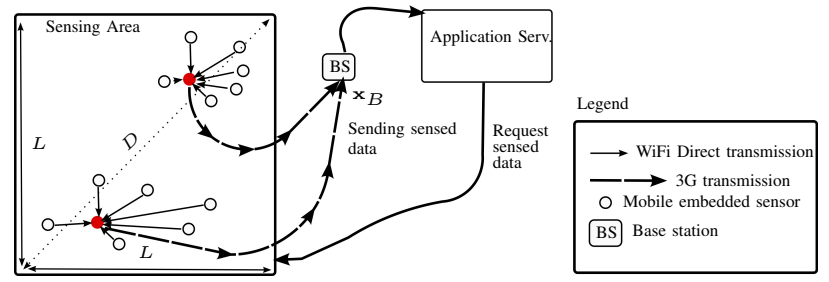

Fig. 1. Applications request sensed data from mobiles with embedded sensors in a $L \times L$ area. Data is collected by one $\mathrm{CH}$ (using WiFiDirect) that compresses the data before offloading it to the closest BS (using 3G), and onto the ACS.

mobility model is incorporated into our evaluation.

Section II compares and contrasts our contribution with the related work. Section III motivates the algorithms. Section IV discusses the algorithm design. Section $\mathrm{V}$ details the experimental setup and our results.

\section{RELATED WORK}

The flexible provisioning of mobile applications due to cloud computing has motivated significant work on the development of platforms for "Mobile Cloud Computing (MCC)." These applications rely on sensed data from embedded sensors on the mobiles. The reliance on the cloud for computation can be costly due to data transmission overheads. Performance degradation may also occur due to limited bandwidth availability. Platforms such as MAUI [4], CloneCloud [3] and ThinkAir [15], seek to balance the trade-off between local processing costs on mobiles and the cost of transferring data to/from data centres. They select parts of the application to offload to the cloud server but do not consider collaboration between mobiles for further energy savings.

iCoMe [29] is an incentive-based cooperative resource management technique that focuses on increasing the revenue of the service provider; mobiles actively download data from available cellular and WiFi connections. In contrast, we consider the case when mobiles offload data to the cloud server for mobile sensing applications. We do not consider the redundancy in the collaboratively offloaded data, and assume that frequent pattern mining [18], Statistical Learning Theory [7] or Rank Reduction [6] do not achieve sufficient reduction. Penner et al. [20] have presented 'Transient Clouds', a cloud-on-the-fly approach for collaboration between mobiles. Serendipity [30] and Mobile Device Cloud [19] utilize connected wireless networks to use the computational resources of other mobiles in close proximity. Similarly, our approach uses collaboration between mobiles to offload data to a cloud server. The crucial difference is that our approach uses the distances between the mobiles and the $\mathrm{BS}$ to select the best $\mathrm{CH}(\mathrm{s})$ to offload the data on behalf of the network. This reduces the number of high cost cellular network transmissions required.

The selection of one/many mobile(s) to report-back sensor data has been widely studied in wireless sensor networks. The routing protocol LEACH [13] stochastically selects CHs. Many $\mathrm{CH}$ selection protocols have adapted the underlying threshold framework of LEACH to improve network lifetime and energy-efficiency with mixed results according to [23]. Factors like residual energy, in [11] and [31], distance to the BS, in [14], and centralized algorithms with location information at the BS, in [12], have been used to study $\mathrm{CH}$ selection. Semantic Clustering Models with fuzzy inference systems have also been introduced [25]. We take a similar approach to LEACH. We consider the challenges presented by using mobiles as a sensing platform. These challenges include the non-static nature of the mobiles, which implies that we must reconfigure the $\mathrm{CH}$ as time evolves. We show that the fact that location information is available in the mobile sensing scenario may be advantageous. We use the non-static nature of the mobiles and location information to optimize the trajectories of the $\mathrm{CHs}$, e.g. chained assignments of $\mathrm{CH}-$ ship, for energy-saving, which is novel. Neighbouring mobiles collaborate by modulating their selection probability relative to a well-positioned $\mathrm{CH}$ to ensure that the $\mathrm{CH}$-ship passes from a well placed position to a better one successively. Our contribution goes significantly beyond previous approaches in that we provide a solution for: allocating $\mathrm{CH}$ responsibility fairly; successively improving $\mathrm{CH}$ selection through time by biasing the thresholds of neighbouring mobiles and then forgetting this bias after a suitable time has elapsed, to save battery life; and finally, allowing the number of $\mathrm{CHs}$ to increase and decrease as the sensing scenario demands.

\section{Problem SET-UP}

We consider the problem of selecting $\mathrm{CHs}$ periodically from a given clustering solution in such a way that the energy consumed during data transmission to an ACS is minimized. The set of mobiles from which the $\mathrm{CHs}$ are selected are mobile handsets and the cellular network is used as the communication medium between the $\mathrm{CH}$ and ACS. Deployments in open areas such a park, a shopping mall, a railway station etc. which lack WiFi are considered in Fig. 1. The ACS requests sensing data from the application client installed on the mobiles. Instead of each mobile sending data, mobiles collaborate, and a reduced number of them send sensing information about the temperature, humidity, percentage of gas etc. in the area of interest to the application. A mobile transfers sensed information using WiFi-Direct [21], [22] to a $\mathrm{CH}$ who is responsible for compressing the data. WiFi-Direct supports one-2-one and one-2-many operations over WiFienabled mobiles but does not require a WiFi access point. This allows peer-2-peer transmissions between the mobiles. We assume that each $\mathrm{CH}$ connects to one cellular BS in its micro-cell and offloads the sensed data.

We consider a duration of $Z$ seconds, which is subdivided into time intervals of $I$ seconds. Sensor readings are taken during each interval, $I$. The k-th time interval is $(k-1) I<$ $t \leq k I$. Each mobile has a unique identifier, $n \in \mathcal{N}$, where $\mathcal{N}$ is the set of mobiles sensing the environment. The mobiles have equal transmission, reception and processing capabilities. The total number of mobiles located within the area of interest (Fig. 1) is denoted $|\mathcal{N}|$. The area to be sensed is assumed to be a square grid with length $L$ meters. The top right coordinates 
of the square is the vector $[L, L]^{T}$. We consider $\mathrm{CH}$ selection in 2-D as opposed to 3-D for notational simplicity. The $\mathrm{n}$-th mobile's coordinates during the $\mathrm{k}$-th time interval is $\mathbf{x}_{n}(k)$. The distance between two mobiles is

$$
d\left(\mathbf{x}_{i}(k), \mathbf{x}_{j}(k)\right)=\left\|\mathbf{x}_{i}(k)-\mathbf{x}_{j}(k)\right\|^{2} .
$$

The decision variable $y_{n}^{k} \in\{0,1\}$ indicates whether the mobile becomes the $\mathrm{CH}$ of a cluster during time interval $k$. If $y_{n}^{k}=1$, mobile $n$ is a $\mathrm{CH}$ for time interval $k$.

Assumption 1: We assume the mobiles can be partitioned into $X$ clusters. Once the $\mathrm{CHs}$ have been selected, the mobiles select the nearest $\mathrm{CH}$ as their $\mathrm{CH}$, and form the set $\mathcal{C}_{i}$-the set of members of the i-th cluster- using a spanning tree, and assumption 1 holds. The sets $\mathcal{C}_{i}, \forall i$ have the following properties: (1) there are no empty clusters, $\mathcal{C}_{i} \neq \emptyset, i \in[1, X]$; (2) the cluster membership does not overlap, $\mathcal{C}_{i} \cap \mathcal{C}_{j}=\emptyset, i, j \in$ $[1, X], i \neq j$; and finally (3) all mobiles are assigned to one cluster, $\bigcup_{i \in[1, X]} \mathcal{C}_{i}=\mathcal{N}$. The $\mathrm{CH}$ of cluster, $\mathcal{C}_{i}$, is responsible for aggregating the sensed data received from the cluster members. Every $I$ seconds, the data aggregated from cluster $C_{i}$ is transferred to the BS. The BS lies at coordinates $\mathbf{x}_{B}$.

Assumption 2: It is assumed that the distance between all mobiles and the BS is greater that the distance between the mobiles that can directly communicate with each other:

$$
d\left(\mathbf{x}_{B}, \mathbf{x}_{n}(k)\right)>d\left(\mathbf{x}_{n}(k), \mathbf{x}_{j}(k)\right), \quad \forall j, n \in \mathcal{N}, \forall k
$$

This assumption is fundamental; the success of our approach depends on the fact that mobile-2-mobile $(\mathrm{m} 2 \mathrm{~m})$ communications are cheaper, in terms of energy usage than mobile-2-BS (m2B) communications. In other words our approach is suited to deployments where the sum of the energy expended by many $\mathrm{m} 2 \mathrm{~m}$ transmissions, costs less than the energy expended by one $\mathrm{m} 2 \mathrm{~B}$ communication. The energy consumed by mobile $n$ in time interval $k$ is due to its support of three functionalities: (1) sensing, which costs $S_{n}^{k}$; (2) transmission of data, which costs $T_{n}^{k}$; and finally, (3) reception of data, either $\mathrm{m} 2 \mathrm{~B}$ or $\mathrm{m} 2 \mathrm{~m}$, which costs $R_{n}^{k}$, in terms of energy usage respectively. The total energy expended by mobile $n$ during interval $k$ is

$$
E_{n}^{k}=S_{n}^{k}+T_{n}^{k}+R_{n}^{k}
$$

The dominant term in this sum is $T_{n}^{k}$, the total energy expended is approximately equal to the transmission $\operatorname{cost} E_{n}^{k} \approx$ $T_{n}^{k}$. The path loss exponent for cellular transmissions, e.g. $\mathrm{m} 2 \mathrm{~B}$ transmissions, is denoted $\lambda$. The energy lost during $\mathrm{m} 2 \mathrm{~B}$ transmissions is proportional to the distance $d\left(\mathbf{x}_{B}, \mathbf{x}_{n}(k)\right)$ raised to the power of $\lambda$, e.g.

$$
T_{n}^{k} \propto d\left(\mathbf{x}_{B}, \mathbf{x}_{n}(k)\right)^{\lambda} \quad \text { if } y_{n}^{k}=1 .
$$

The property that we exploit to decrease energy usage is described as follows. We drop proportionality factors and offsets in favour of a making a simplified argument, e.g. instead of using $T_{n}^{k}=a+b d\left(\mathbf{x}_{B}, \mathbf{x}_{n}(k)\right)^{\lambda}$ we let $a=0$ and $b=1$ to simplify the notation in this motivation section, using $T_{n}^{k}=d\left(\mathbf{x}_{B}, \mathbf{x}_{n}(k)\right)^{\lambda}$. Referring to (Eqn. 2), the crucial point is that the marginal cost of the two types of transmission,

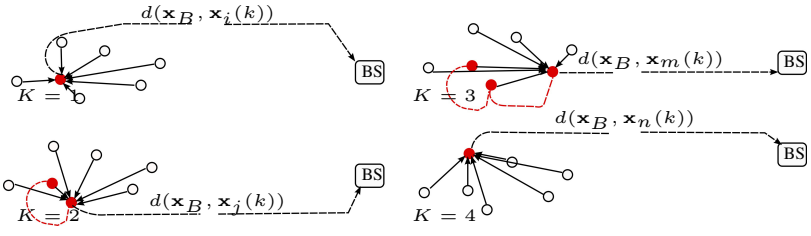

Fig. 2. CH Trajectories: $\mathrm{CHs}$ (red dots) and transmissions between the mobiles and the $\mathrm{CH}$ (solid black arrows) are illustrated at times $k=1,2,3$ and 4 . The transmission between the $\mathrm{CH}$ at each time to the BS is illustrated with a dashed black arrow, and $d\left(\mathbf{x}_{B}, \mathbf{x}_{q}(k)\right)$ meters. The inter-mobile distance is small relative to the $\mathrm{CH}-2$-BS distance. For $k=1,2,3$ the $\mathrm{CH}$ follows a trajectory (dashed red line), which moves the $\mathrm{CH}$ successively closer to the $\mathrm{BS}$, reducing the cost of the $\mathrm{CH}-2-\mathrm{BS}$ transmission. At $k=4$ the $\mathrm{CH}$ jumps to a new location. For $k=1,2,3$ energy is saved. At time $k=4$ less energy is saved, $m_{n}$ is smaller, but fairness is preserved.

$\mathrm{m} 2 \mathrm{~m}$ and $\mathrm{m} 2 \mathrm{~B}$, grows rapidly as a function of the transmission distances, raised to the exponent $\lambda$,

$$
m_{n}=\left|d\left(\mathbf{x}_{B}, \mathbf{x}_{n}(k)\right)^{\lambda}-d\left(\mathbf{x}_{n}(k), \mathbf{x}_{j}(k)\right)^{\lambda}\right|
$$

We save $m_{n}$ when mobile $n$ performs a local transmission to mobile $j$ instead of a transmission to the BS. The greater the average distance of the mobiles from the BS, $\frac{1}{|\mathcal{N}|} \sum_{n \in \mathcal{N}} d\left(\mathbf{x}_{B}, \mathbf{x}_{n}(k)\right)$ the greater the gain in energy saving we expect. In the worst case scenario, all mobiles communicate directly with the BS at a cost $\sum_{n \in \mathcal{N}} d\left(\mathbf{x}_{B}, \mathbf{x}_{n}(k)\right)^{\lambda}$. Considering the simple case $X=1$, in the best case scenario, all mobiles communicate with one $\mathrm{CH}, j$, who transmits all of the sensed data to the BS, at a cost $d\left(\mathbf{x}_{B}, \mathbf{x}_{j}(k)\right)^{\lambda}$. The total saving for each mobile that does not communicate with the BS is $\sum_{n \in \mathcal{N} \backslash j} m_{n}$. Let $s(j)=d\left(\mathbf{x}_{B}, \mathbf{x}_{j}(k)\right)^{\lambda}+$ $\sum_{n \in \mathcal{N} \backslash j} d\left(\mathbf{x}_{n}(k), \mathbf{x}_{j}(k)\right)^{\lambda}$. The upper bound, $u$, on the potential energy saving, when $X=1$, for a given $n$ is the difference between the worst and best case scenarios above:

$$
u=\sum_{n \in \mathcal{N}} d\left(\mathbf{x}_{B}, \mathbf{x}_{n}(k)\right)^{\lambda}-\min _{j} s(j)
$$

which gives a good back-of-the-envelope estimate of how much energy this technique could save. The upper bound $u$, for $X=1$, is constructed from the energy cost of the worst case, where all mobiles transmit to the BS, and the alternative, which is where the best mobile is chosen to be the $\mathrm{CH}$. The lower bound is 0 . To ensure that assumption 2 holds we assert that the set of mobiles that lie within the distance $d$, a user defined constant, of $\mathrm{CH}, n$, is denoted $\mathcal{W}_{n}$. We bound the maximum distance of $\mathrm{m} 2 \mathrm{~m}$ transmissions so that energy savings are achieved with high probability. A less exact, simplified upper bound follows

$$
\sum_{n \in \mathcal{N}} d\left(\mathbf{x}_{B}, \mathbf{x}_{n}(k)\right)^{\lambda}-\min _{j}\left(|\mathcal{N} \backslash j| d^{\lambda}+d\left(\mathbf{x}_{B}, \mathbf{x}_{j}(k)\right)^{\lambda}\right)
$$

CH Trajectory Minimization: Taking this analysis one step further, and considering the simple case of $X=1 \mathrm{CHs}$, a greedy mechanism for reducing energy consumption, if the location of the mobiles is known, is to consider the formation of chains of $\mathrm{CH}$ heads through time, where each successively 


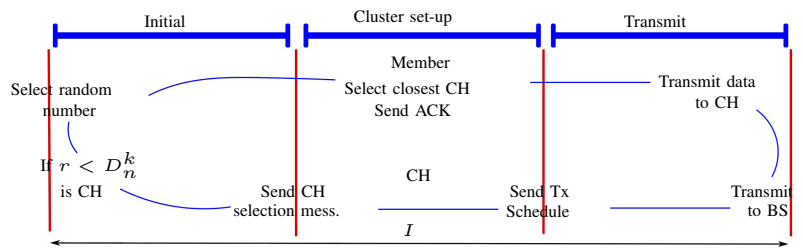

Fig. 3. Each interval $I$ consists of an initial, cluster set-up and transmit phase. $\mathrm{CHs}$ are selected based on a random trial in the initial phase. The $\mathrm{CH}$ announces its selection and the other mobiles select the closest $\mathrm{CH}$ as their $\mathrm{CH}$ during the cluster set-up phase. The $\mathrm{CH}$ sends a transmit schedule and the mobiles transmit sensing data to the $\mathrm{CH}$ during the transmit phase. Finally, the $\mathrm{CH}$ transmits the aggregated data to the $\mathrm{BS}$.

chosen $\mathrm{CH}$ reduces the energy cost of transmission to the $\mathrm{BS}$, e.g. by selecting mobiles $i, j$, and $m$ and so on, such that

$$
d\left(\mathbf{x}_{B}, \mathbf{x}_{i}(k)\right)>d\left(\mathbf{x}_{B}, \mathbf{x}_{j}(k+1)\right)>d\left(\mathbf{x}_{B}, \mathbf{x}_{m}(k+2)\right) \ldots
$$

This procedure is illustrated in Fig. 2. The $\mathrm{CH}$ is moved closer to the $\mathrm{BS}$ as $k$ increases, which is far away from the mobiles; this movement is called the trajectory of the $\mathrm{CHs}$ and it is illustrated in red. It does not involve the physical movement of any of the mobiles, but a change in the role that they perform. The problem with such an approach is that when the $\mathrm{CH}$ has moved as close as possible to the $\mathrm{BS}$, it cannot move closer, and the mobile's battery becomes depleted as this mobile is unfairly sending the costly offload transmissions to the BS. In the next section we describe an algorithm that stochastically selects the $\mathrm{CH}$ in such a way that the $\mathrm{CH}$-ship may jump out of its current trajectory and start a new trajectory at a new position. This is illustrated in Fig. 2 at time $k=4$. A new mobile is selected as the $\mathrm{CH}$, which has no relation with previous CHs. This jump spreadsout the costly transmissions to the BS between the mobiles, introducing fairer $\mathrm{CH}$ selection.

The sensed data available in mobile $n$ is $V_{n}$ bits. The data available for transmission after aggregation in the $\mathrm{CH}$ is

$$
V_{i}=c \sum_{\forall n \in C_{i}} V_{n} \text { bits. }
$$

We make the assumption that the aggregation factor is on average $c$ and that the accuracy of the technique is not significantly affected by different $\mathrm{CH}$ selections. Finally, if the battery level of mobile $n$ at the start of time interval $k$, denoted $b_{n}^{k}$, is below $\gamma$ of the full battery level $B_{n}$, the mobile may have to be powered off so that the battery of one mobile dying does not affect the sensing solution, $b_{n}^{k}>=\gamma B_{n}, \forall n \in \mathcal{N}, \forall k \in[0, Z / I]$. This restriction is imposed on all mobiles for every time interval to ensure battery availability for sensing, transmission and receiving. At the end of each interval, the battery level is checked

$$
b_{n}^{k+1}=b_{n}^{k}-S_{n}^{k}-T_{n}^{k}-R_{n}^{k} .
$$

\section{Stochastic, Fair Cluster-Head Selection}

We stochastically select the $\mathrm{CH}$ for the next time interval, $k+1$, using a fair, greedy algorithm, which aims to (1) optimize the energy usage of each current $\mathrm{CH}$ in a collaborative way and (2) distribute the transmission costs among the mobiles, in a manner which is aligned with the $\mathrm{CH}$ trajectory minimization illustrated in Fig. 2.

Fairness: A threshold value, $D_{n}^{k}$ for mobile $n$, for the k-th time interval, is used to determine the probability that mobile $n$ becomes the $\mathrm{CH}$. Each mobile randomly draws a number, $r$, between 0 and 1 and compares the outcome of the trial, $r$, with the threshold, $D_{n}^{k}$. The decision rule for determining the $\mathrm{CH}$-ship of a mobile $n$ at time $k$ is

$$
y_{n}^{k}= \begin{cases}0, & \text { if } r \geq D_{n}^{k}, \\ 1, & \text { if } r<D_{n}^{k} .\end{cases}
$$

Information about the distance of the $n$-th mobile from the $\mathrm{CH}$ is not incorporated in this decision rule -all mobiles use the same threshold to determine whether or not they become the $\mathrm{CH}$ during the next time interval. This process is fair in that all mobiles are equally likely of becoming the $\mathrm{CH}$ during the next time interval. The threshold $D_{n}^{k}$ determines the expected number of $\mathrm{CHs}$ during the $\mathrm{k}$-th time interval. The parameter, $p$, sets the probability that a given mobile will be a $\mathrm{CH}$; the threshold trace is a periodic function.

$$
D_{n}^{k}=B=\frac{p}{1-p \bmod \left(k, p^{-1}\right)}, \quad \forall n \in \mathcal{N}, \forall k
$$

Fig. 4, row 1, describes the evolution of 5 cycles of this base threshold, $B$ as a function of time. The parameter $p$ sets the minimum value this cyclic function achieves. The larger the $p$, the greater the number of mobiles that will be selected as $\mathrm{CH}$. The crucial point is that each mobile traces out exactly the same threshold evolution because $p$ is the same for all mobiles. LEACH operates in this manner. The difference between our algorithm and LEACH is that (1) we allow a mobile to become a $\mathrm{CH}$ multiple times; (2) we modulate the threshold, the effective $p$, based on location information; (3) we adapt and forget the threshold modulation depending on the mobility of the mobiles; (4) we allow the number of $\mathrm{CHs}$ to fluctuate so that the number of $\mathrm{CHs} X$ can change with time. Mechanisms for achieving these functionalities are described. Threshold Modulation: We propose two algorithms that modulate the threshold of certain mobiles depending on the distance between the $\mathrm{CH}$ of the previous interval and the $\mathrm{BS}$. alg1 alters the threshold for the nearest neighbours, $\mathcal{W}_{i}$, of the $\mathrm{CH}, i$, as a function of their distance to the BS. We modulate the threshold in (Eqn. 12) in the decision rule (Eqn. 11) of a mobile $n \in \mathcal{W}_{i}$ by including a distance function factor $f\left(\mathbf{x}_{B}, \mathbf{x}_{n}(k)\right)=\frac{1}{2}\left(\frac{D-d\left(\mathbf{x}_{B}, \mathbf{x}_{n}(k)\right)}{D B}+1\right)$ in the threshold trace. The constant $D$ is the maximum permissible $\mathrm{m} 2 \mathrm{~m}$ communication distance, a user defined variable. A good choice in Fig. 1 is $D=\sqrt{2 L^{2}}$. The term, $S=\max f\left(\mathbf{x}_{B}, \mathbf{x}_{n}(k)\right) B$, is the maximum value the distance function, $f$ can achieve for a given position $\mathbf{x}_{n}(k)$. Every time the mobile moves, $f$ and $S$ are updated.

$$
D_{n}^{k}= \begin{cases}\frac{B}{2 S}\left(\frac{D-d\left(\mathbf{x}_{B}, \mathbf{x}_{n}(k)\right)}{D B}+1\right) & \forall n \in \mathcal{W}_{i} \\ B & \forall n \in \mathcal{N} \backslash \mathcal{W}_{i}\end{cases}
$$


The advantage of including $\frac{f}{S}$ is that greater energy is saved with high probability as $\mathrm{CH}$ trajectories, like the trajectory in (Eqn. 8), are followed. The short-coming of this approach is that the algorithm is not as fair as the LEACH approach (Eqn. 12). Mobiles with modulated thresholds may become the $\mathrm{CH}$ too frequently, which exhausts their battery-life unfairly. alg2: We incorporate an additional forgetting factor $g$ into the threshold trace generation function in (Eqn. 13). The role of $g$ is to ensure that after a few intervals of not being chosen to be a $\mathrm{CH}$, mobile $n$ stops modulating its threshold using $f / S$.

$$
g(k)=\left(\frac{1}{(k-v+1)^{l}}\right) \text { when } n \in \mathcal{W}_{i}
$$

The time when mobile $n$ starts modulating its threshold by $f / S$ is denoted $v$. The power in the denominator $l$ sets the rate at which the mobile forgets. In Fig. 4 we use $l=.8$ and $v=$ 100 , which implies that a neighbouring mobile was elected $\mathrm{CH}$ at time $k=99$. The choice $l=.8$ means the effect of $f$ is forgotten after 30 samples. Multiplying $g$, a decay function by the modulation factor $f$ causes the effect of the modulation factor to be forgotten. We set $S=\max f\left(\mathbf{x}_{B}, \mathbf{x}_{n}(k)\right) g(k)$.

$$
D_{n}^{k}= \begin{cases}B f\left(\mathbf{x}_{B}, \mathbf{x}_{n}(k)\right) g(k), & \forall n \in \mathcal{W}_{i}, \\ \text { if } B \leq B f\left(\mathbf{x}_{B}, \mathbf{x}_{n}(k)\right) g(k) . & \text { otherwise } \\ B & \forall n \in \mathcal{N} \backslash \mathcal{W}_{i}\end{cases}
$$

Once this new threshold value becomes less than the base threshold, the mobile no longer modulates the threshold by $f / S$, unless the mobile is in the neighbourhood of a $\mathrm{CH}$ at some future time. Fig. 4 depicts the effects of the different threshold algorithms. After a mobile becomes the $\mathrm{CH}$, it establishes a one-2-many connections with its peers and sends its self-election information along with its position coordinates using WiFi-Direct. The second algorithm, alg2, introduces an additional forgetting mechanism for each mobile that was in the neighbourhood of the $\mathrm{CH}, i$, e.g. the set $\mathcal{W}_{i}$ in an previous interval, but that was not selected to be a $\mathrm{CH}$. The forgetting mechanism allows mobiles to forget about: (1) location information if it may be outdated; (2) previous $\mathrm{CH}$ assignments so that after a suitable time has elapsed the mobile uses the fair threshold generating rule (in Eqn. 12).

Control of flow: $\operatorname{alg} 1$ and alg2 are divided into three phases in Fig. 3: Initial, Cluster-Setup and Transmit. They cycle through these phases to select each new $\mathrm{CH}$ every $I$ seconds.

Initial Phase: In this phase, the $\mathrm{CHs}$ for the interval are selected in a distributed way. Each mobile selects a uniformly distributed random variable $r$ in the range $[0,1]$. If this number is less than the threshold defined for the interval, $D_{n}^{k}$, the mobile becomes a $\mathrm{CH}$ for that time interval (Eqn. 11). The base-threshold is used to initiate the thresholds during the first interval, $k=1$. If mobile $n$ is a $\mathrm{CH}$ during interval $k>1$, the thresholds of the members of the set $\mathcal{W}_{n}$ are modulated by the distance function $f / S$ if $\operatorname{alg} 1$ is used, or the distance function and the forgetting factor $f / S$ and $g$ if alg2 is used. Therefore, the outcome of future stochastic trials is biased by the identity of the $\mathrm{CH}$ during the previous interval $n$ and the distance of the nodes in the set $\mathcal{W}_{n}$ from the BS. Mobiles that are not members of the $\mathcal{W}_{n}$, or the neighbour sets of other CHs use the base-threshold $B$ to perform $\mathrm{CH}$ selection trials.
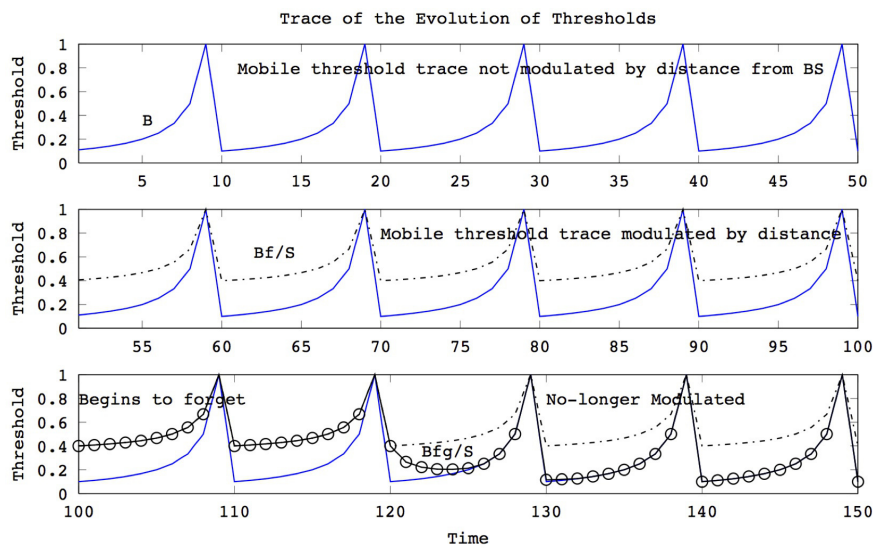

Fig. 4. The threshold trace (solid line, row 1), $B$, for intervals $1 \leq k \leq 50$ (Eqn. 12). At time $51 \leq k \leq 100$ the distance function is incorporated into the trace (Eqn. 13), dashed-dot line, row 2. The minimum value of the threshold hold is modulated by the distance of the mobile from the BS. In row 3 (o-solid line), $101 \leq k \leq 150$ the forgetting factor (Eqn. 15) removes the effect of the distance function from the threshold by time $k=130$.
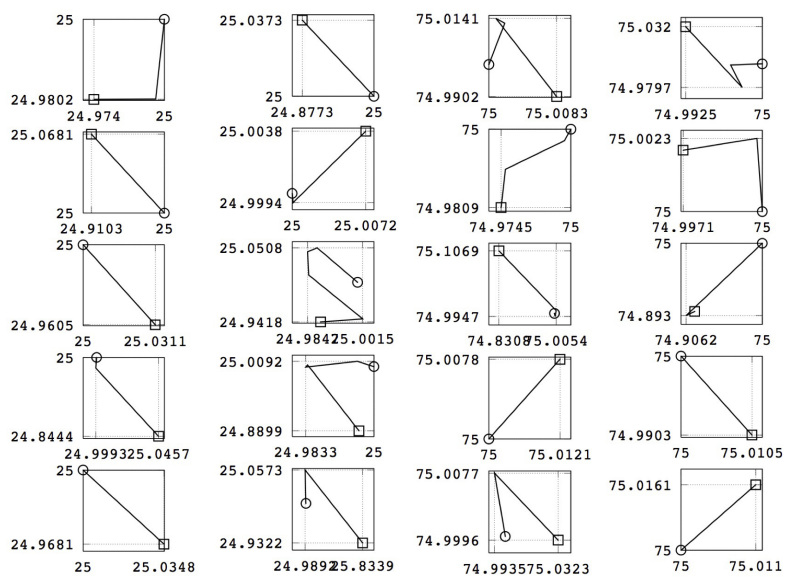

Fig. 5. Truncated Levy Walk Mobility Patterns for ten instances of mobiles starting at $[25,25]^{T}$ (columns 1,2) and $[75,75]^{T}$ (columns 3,4) for two hours.

Cluster Setup Phase: Each non-CH mobile must choose to belong to one $\mathrm{CH}$. Due to the mobility of the mobiles, the $\mathrm{CH}$ chosen is the closest (using Eqn. 1) $\mathrm{CH}$ during that timeinterval. Each mobile predicts its mobility path in the timeinterval $k$ and the $\mathrm{CH}$ with the closest $\mathrm{CH}$ is selected for that time-interval. An acknowledgement is then sent to the selected $\mathrm{CH}$ to inform it about its new member.

Transmit Phase: The $\mathrm{CH}$ receives all mobile messages and creates a transmission schedule for all of its member mobiles. The members transmit the sensed data during their allocated time. When the data is received, the $\mathrm{CH}$ compresses the data into a single data stream and transmits it to the BS.

\section{Evaluation}

We assess (1) the gain in energy saved over LEACH and (2) the distribution of energy saving gains over all mobiles participating in sensing. To achieve this, we vary the number of mobiles, the probability $p$ of the each mobile becoming a $\mathrm{CH}$, and the slope of the forgetting factor $l$. 

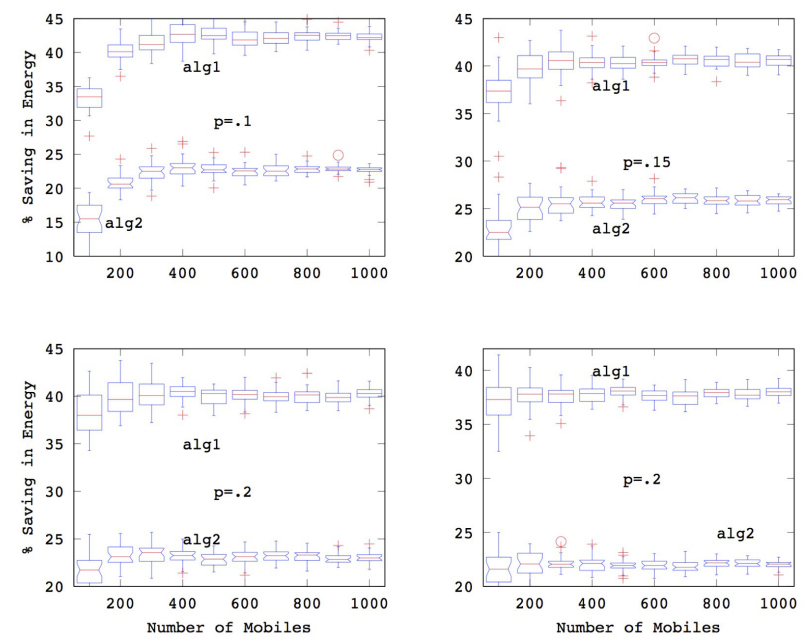

Fig. 6. Energy gain of alg1 and alg2 over LEACH

Simulation Model: The following simulation set-up is used to compare the performance of alg1 and alg2 with LEACH. The sensing area has dimensions $L \times L$ where $L=100 \mathrm{~m}$. The BS is at location $\mathbf{x}_{B}=[500,500]^{T}$. The time interval between $\mathrm{CH}$ selections is of duration $I=120 \mathrm{~s}$. Each experiment is run for a duration of $Z=14400 \mathrm{~s}$. Each mobile device's movement is modelled using the truncated Levy walk mobility model presented in [24] which can be represented by flight length $\varphi$, flight direction $\theta$, flight time $t_{f}$ and pause time $t_{p}$. Our model picks the flight length and pause times randomly from Levy distributions with stability exponents $\alpha=1.5$ and $\beta=0.5$ respectively. The flight direction $\theta$ follows a uniform distribution and flight time is calculated using a constant speed of $1 \mathrm{~m} / \mathrm{s}$ during the flight. The position of the mobile is updated every 40 s, 3 times per time-interval $I$. We select $0.01 \mathrm{~m}$ and $1000 \mathrm{~s}$ respectively for the scale parameters of the flight length and pause times Levy distributions. These values are motivated by the fact that the mobile devices are within a mean value of one meter from their original position every time a time interval of 120 seconds has elapsed. For the purpose of illustration, samples from the levy walk model for ten instances of mobiles, starting at two sets of coordinates $\mathbf{x}_{i}(0)=[25,25]^{T}$ and $\mathbf{x}_{j}(0)=[75,75]^{T}$, and running for a period of two hours is depicted in Fig. 5. The start locations are denoted by circles and the end locations are given by squares. The mobiles have a maximum battery of $5 \mathrm{Wh}$ which decreases over time due to (1) the general usage of the mobile, for the operation of the sensors, and (2) transmitting sensed data. Sensirion offers humidity and temperature sensors for mobiles with an energy consumption of $0.01152 \mathrm{~J} / \mathrm{h}$ [28]. This is taken to be the energy loss associated with sensing. For energy transmission calculations, we adopt the COST-231 propagation model based on the Walfish-Ikegami model for micro-cell deployments [5], [27]. We use the non-line-of-sight path loss model for $2 \mathrm{GHz}$ which is $35.7+38 \log _{10}(d)$. The mobile also uses energy to maintain connections, $0.02 \mathrm{~J} / \mathrm{sec}$ for the cellular network $(3 \mathrm{G})$ and $0.05 \mathrm{~J} / \mathrm{sec}$ for WiFi [2]. We use this maintenance energy cost for WiFi Direct communications.
The number of mobiles sensing the environment is varied in steps of 100 from 100 to 1000 . Each experiment is randomly initialized and run 30 times. The probability of a $\mathrm{CH}$ being selected, $p$, is examined to study how different probabilities $p \in\{.1, .15, .2, .25\}$ affect energy savings. We compare LEACH with alg1 and alg2 in Fig. 6, and compute box-plots for the results for each $p$ and number of mobiles pair. We vary the probability of $\mathrm{CH}$ selection $p$ for different numbers of mobiles. We conclude that: (1) both alg1 and alg2 improve the gains in energy savings over LEACH (by $\approx 40 \%$ and $\approx 20 \%$ ) irrespective of the number of mobiles and the probability $p$. (2) As expected, the variation in the energy gains decreases as the number of mobiles increases. (3) Alg1 achieves better gains than alg2; however, the number of mobiles used as $\mathrm{CHs}$ is greater for alg1 than alg2. This is explained by considering the role of the slope of the forgetting factor $l$. (4) Alg1 and alg2 are fair, in that remarkably, the inter-quartile range of the energy saving (over LEACH) is $\approx 1 \%$. This implies that most devices achieve an energy saving which is within 1 or 2 $\%$ or the mean energy saving. (5) In terms of the role of the probability $p$ in determining the percentage energy saving, the gain in energy saving over LEACH decreases as the probability of each mobile being a $\mathrm{CH}$ increases.

We consider now the effect of the slope of the forgetting factor in Table I, e.g. for alg2. As the parameter of the forgetting factor slope $l$ increases, we expect that alg2 will forget slower. We re-run the experiments above for $l=.6$ and .9 to determine the role of the forgetting factor $l$. Table I tabulates the mean energy gain for alg2 over LEACH. The standard deviation of the gain is also illustrated. Once again, the deviation is small relative to the mean energy gain -the deviation is approximately $1 \%$ again. Table I confirms that as $l$ is increased from $.6 \rightarrow .8 \rightarrow .9$ the average gain is decreased. The mobiles forget slower, and energy saving gains are reduced. The overall trend is that increasing $p$ increases the energy saving gains for $p=.15$, but energy saving gains then decrease as $p$ increases further. The fact that the best gains are achieved for a probability $p=.15$ and $l=.6$ motivates the need for a more in-depth study that determines the best $p, l$ pairs for different deployments. One interesting observation is how the number of $\mathrm{CHs}$ changes over time for alg1 and agl2. A secondary study on the effect of $p, l$ on the number of $\mathrm{CHs}$ would help refine the deployment of alg1 and alg2 in different scenarios where different numbers of $\mathrm{CHs}$ were preferable. The ability of these algorithms to select different numbers of mobiles to be $\mathrm{CHs}$ is advantageous, because irrespective of the number of CHs, $X$, we achieve significant energy savings.

Future Implementation Outlook: Our approach assumes that the participating mobiles have location information (using GPS) and knowledge of the location of the BS. This assumption is reasonable for android operating systems. Details like cell id, location area code, Mobile Country Code, Mobile Network Code are available to applications [1]. In addition, several public databases like OpenCell [10] contain a database of all BSs and are used by applications today. 
TABLE I

MEAN \% GAIN (STANDARD DEVIATION IN BRACES)

\begin{tabular}{c|c|c|c|c|c}
\hline & Mobiles & $p=.1$ & $p=.15$ & $p=.2$ & $p=.25$ \\
\hline \multirow{5}{*}{$l=.6$} & 100 & $16.2(2.15)$ & $23.3(2.17)$ & $22.1(1.68)$ & $21.9(1.61)$ \\
& 200 & $22.3(1.48)$ & $25.5(1.24)$ & $23.4(1.44)$ & $22.4(1.04)$ \\
& 300 & $23.0(1.42)$ & $26.4(1.26)$ & $23.6(1.15)$ & $22.3(0.74)$ \\
& 400 & $23.9(1.33)$ & $25.9(0.76)$ & $23.8(0.73)$ & $22.3(0.61)$ \\
& 500 & $23.2(1.28)$ & $25.9(0.9)$ & $23.4(0.57)$ & $22.2(0.55)$ \\
& 600 & $23.1(1.06)$ & $26.3(0.76)$ & $23.5(0.76)$ & $22.3(0.54)$ \\
& 700 & $23.4(0.82)$ & $26.4(0.6)$ & $23.7(0.63)$ & $22.3(0.62)$ \\
& 800 & $23.5(0.74)$ & $26.3(0.66)$ & $23.5(0.68)$ & $22.4(0.49)$ \\
& 900 & $23.6(0.59)$ & $26.3(0.68)$ & $23.5(0.53)$ & $22.4(0.44)$ \\
& 1000 & $23.3(0.68)$ & $26.2(0.55)$ & $23.6(0.48)$ & $22.4(0.52)$ \\
\hline \hline \multirow{5}{*}{$l=.9$} & 100 & $11.1(2.13)$ & $18.3(2.17)$ & $16.3(1.51)$ & $16.2(1.42)$ \\
& 200 & $15.6(1.28)$ & $19.7(1.33)$ & $17.4(0.94)$ & $16.2(1)$ \\
& 300 & $16.7(1.26)$ & $20.4(1.32)$ & $17.6(1.03)$ & $16.5(0.71)$ \\
& 400 & $17.1(1.37)$ & $20.3(0.69)$ & $17.8(0.65)$ & $16.5(0.64)$ \\
& 500 & $17.3(0.9)$ & $20.3(0.81)$ & $17.2(0.75)$ & $16.2(0.52)$ \\
& 600 & $16.8(0.98)$ & $20.5(0.83)$ & $17.6(0.67)$ & $16.4(0.48)$ \\
& 700 & $17.2(0.68)$ & $20.9(0.74)$ & $17.9(0.67)$ & $16.6(0.52)$ \\
& 800 & $17.3(0.7)$ & $20.6(0.54)$ & $17.8(0.62)$ & $16.6(0.41)$ \\
& 900 & $17.1(0.51)$ & $20.6(0.54)$ & $17.5(0.54)$ & $16.4(0.53)$ \\
& 1000 & $16.9(0.54)$ & $20.5(0.5)$ & $17.5(0.5)$ & $16.3(0.42)$ \\
\hline
\end{tabular}

\section{CONCLUSiOnS}

This paper focuses on reducing the energy-consumption when sensed information is offloaded to an ACS. When there are no $\mathrm{WiFi}$ access points in close proximity, mobiles rely on the cellular network to transfer the sensed data, which is costly in terms of energy usage. We introduce a collaborative approach for mobiles, that efficiently selects well-placed $\mathrm{CHs}$ to make the high energy transmission to the BS. Collaboration is based on WiFi-Direct, which allows peer-2-peer transmissions between the mobiles. Our approach is novel in that it uses the current distance (and potential future distances) between a mobile (and it neighbours) and the BS to select a $\mathrm{CH}$ to offload data to, and to bias future selection decisions. These results have real-benefits for practitioners: we show energy savings in the range of 20 to $40 \%$ over the state-of-the-art are possible.

\section{REFERENCES}

[1] Android Developers. CellLocation.

[2] Niranjan Balasubramanian, Aruna Balasubramanian, and Arun Venkataramani. Energy Consumption in Mobile Phones: A Measurement Study and Implications for Network Applications. In Proc. 9th ACM SIGCOMM Conf. on Int. Meas. Conf., IMC, pages 280-93, USA, 2009.

[3] Byung Gon Chun, Sunghwan Ihm, Petros Maniatis, Mayur Naik, and Ashwin Patti. CloneCloud: elastic execution between mobile device and cloud. In Proc. 6th ACM Conf. Comp. Sys., EuroSys, pages 301-14, USA, 2011.

[4] Eduardo Cuervo, Aruna Balasubramanian, Dae Ki Cho, Alec Wolman, Stefan Saroiu, Ranveer Chandra, and Paramvir Bahl. MAUI: making smartphones last longer with code offload. In Proc. 8th ACM Int. Conf. on Mobi. Sys., App., and Ser., pages 49-62, USA, 2010.

[5] Eraldo Damosso and COST Telecom Secretariat. Digital mobile radio towards future generation systems: Cost 231 final report., 1999.

[6] Ruairí de Fréin. Formal concept analysis via atomic priming. In Formal Concept Analysis, volume 7880 of LNCS, pages 92-108. Springer, 2013.

[7] Ruairí de Fréin. Ghostbusters: A parts-based NMF algorithm. In Signals and Systems Conference (ISSC 2013), 24th IET Irish, pages 1-8, 2013.

[8] Ruairí de Fréin. Learning and storing the parts of objects: IMF. In Mach. Learn. for Sig. Proc. (MLSP), IEEE Int. Wkshp, pages 1-6, Sept 2014.

[9] Ruairí de Fréin. Quantized nonnegative matrix factorization. In Dig. Sig. Proc., 19th Int. Conf., pages 377-382, Aug 2014.

[10] ENAikoon. OpenCellID.
[11] M.J. Handy, M. Haase, and D. Timmermann. Low energy adaptive clustering hierarchy with deterministic cluster-head selection. In Proc. 4th Int. Wk. Shp. on Mobi. and Wireless Comm. Net., pages 368-72, 2002.

[12] W.B. Heinzelman, A.P. Chandrakasan, and H. Balakrishnan. An application-specific protocol architecture for wireless microsensor networks. IEEE Trans. Wireless Comm., 1(4):660-70, 2002.

[13] W.R. Heinzelman, A. Chandrakasan, and H. Balakrishnan. Energyefficient communication protocol for wireless microsensor networks. In Proc. 33rd Annu. Hawaii Int. Conf. on Sys. Sci., 2000.

[14] S.H. Kang and Thinh Nguyen. Distance Based Thresholds for Cluster Head Selection in Wireless Sensor Networks. IEEE Comm. Lett. 16(9):1396-9, 2012

[15] Sokol Kosta, Andrius Aucinas, Pan Hui, Richard Mortier, and Xinwen Zhang. Thinkair: Dynamic resource allocation and parallel execution in the cloud for mobile code offloading. In Proc. IEEE INFOCOM, pages 945-53, 2012.

[16] K. Kumar and Yung Hsiang Lu. Cloud computing for mobile users: Can offloading computation save energy? Computer, 43(4):51-6, April 2010.

[17] N.D. Lane, E. Miluzzo, Hong Lu, D. Peebles, T. Choudhury, and A.T. Campbell. A survey of mobile phone sensing. IEEE Comm. Mag., 48(9):140-50, 2010.

[18] Radhika Loomba, Lei Shi, Brendan Jennings, Roy Friedman, John Kennedy, and Joe Butler. Information Aggregation for Mobile Sensing in Mobile Cloud Computing. In Proc. 2nd IEEE Int. Conf. on Mob. Cloud Comp., Serv., and Eng. (MobiCloud), pages 149-58, 2014.

[19] Abderrahmen Mtibaa, Afnan Fahim, Khaled A. Harras, and Mostafa H. Ammar. Towards Resource Sharing in Mobile Device Clouds: Power Balancing Across Mobile Devices. In Proc. 2nd ACM SIGCOMM Wk. Shp. on Mob. Cloud Comp., MCC, pages 51-6, USA, 2013.

[20] T. Penner, A. Johnson, B. Van Slyke, M. Guirguis, and Qijun Gu. Transient clouds: Assignment and collaborative execution of tasks on mobile devices. In Proc. IEEE Global Comm. Conf. (GLOBECOM), pages 2801-6, 2014.

[21] A. Pyattaev, K. Johnsson, S. Andreev, and Y. Koucheryavy. 3GPP LTE traffic offloading onto WiFi Direct. In Proc. IEEE Wireless Comm. and Net. Conf. Wk Shp. (WCNCW), pages 135-40, 2013.

[22] A Pyattaev, K. Johnsson, S. Andreev, and Y. Koucheryavy. ProximityBased Data Offloading via Network Assisted Device-to-Device Communications. In Proc. 77th IEEE Veh. Tech. Conf. (VTC Spring), pages $1-5,2013$.

[23] K. Ramesh and Dr K. Somasundaram. A comparative study of clusterhead selection algorithms in wireless sensor networks. Int. J. of Comp. Sci. \& Eng. Sur., 2(4):153-64, November 2011.

[24] Injong Rhee, Minsu Shin, Seongik Hong, Kyunghan Lee, Seong Joon Kim, and Song Chong. On the levy-walk nature of human mobility. IEEE/ACM Trans. Netw., 19:630-43, 2011.

[25] Atslands R. Rocha, Luci Pirmez, Flvia C. Delicato, rico Lemos, Igor Santos, Danielo G. Gomes, and Jos Neuman de Souza. WSNs Clustering Based on Semantic Neighborhood Relationships. Computer Networks, 56(5):1627-645, 2012.

[26] Alexey Rudenko, Peter Reiher, Gerald J. Popek, and Geoffrey H. Kuenning. Saving portable computer battery power through remote process execution. SIGMOBILE Mob. Comp. and Comm. Rev., 2(1):1926, 1998.

[27] T.K. Sarkar, Zhong Ji, Kyungjung Kim, A. Medouri, and M. SalazarPalma. A survey of various propagation models for mobile communication. IEEE Ant. and Prop. Mag., 45(3):51-82, 2003.

[28] Sensirion. Sht2x - digital humidity \& temperature sensor $(\mathrm{rh} / \mathrm{t})$

[29] H. Shah-Mansouri and V.W.S. Wong. iCoMe: A novel incentivized cooperative mobile resource management mechanism. In Proc. IEEE Global Comm. Conf. (GLOBECOM), pages 4996-5001, December 2014.

[30] Cong Shi, Vasileios Lakafosis, Mostafa H. Ammar, and Ellen W. Zegura. Serendipity: Enabling Remote Computing Among Intermittently Connected Mobile Devices. In Proc. 13th ACM Int. Symp. on Mob. AdHoc Net. and Comp., MobiHoc, pages 145-54, USA, 2012.

[31] M.C.M. Thein and T. Thein. An Energy Efficient Cluster-Head Selection for Wireless Sensor Networks. In Proc. Int. Conf. on Intel. Sys. Modelling and Sim. (ISMS), pages 287-91, 2010.

This research was funded by the: (1) Irish Research Council (IRC) Enterprise Partnership Scheme Postgraduate Research Scholarship, co-funded by Intel Labs, Europe (grant no. EPSPG/2012/407); (2) IRC and Marie Curie 
Actions via grant no. ELEVATEPD/2014/62; (3) the European Commission via the FP7 IAPP project SOLAS (grant no. 612480) and 4) Science Foundation Ireland (and TEAGASC) via grant nos. 13/RC/2077 (and 13/IA/1977). 